\section{REVIEW ARTICLE}

\author{
B.A. Standish \\ J. Spears \\ T.R. Marotta \\ W. Montanera \\ V.X.D. Yang
}

\title{
Vascular Wall Imaging of Vulnerable Atherosclerotic Carotid Plaques: Current State of the Art and Potential Future of Endovascular Optical Coherence Tomography
}

\begin{abstract}
SUMMARY: As stroke is one of the leading causes of death and long-term morbidity worldwide, the research community has studied cardiac embolic sources, as well as vessel wall pathologies. For the latter, attention has been focused on defining morphologic tissue features associated with catastrophic events stemming from the carotid artery. Multiple noninvasive imaging modalities are currently being used to image and classify carotid atherosclerotic plaques, such as MR imaging, CT, and sonography, in an effort to provide clinically relevant predictive metrics for use in patient risk stratification and to define appropriate treatment options. This article compares and contrasts these existing clinical imaging modalities along with discussion of a new endovascular technique originally developed for cardiology, OCT, with which 3D comprehensive high-resolution images of the arterial wall can be acquired.
\end{abstract}

ABBREVIATIONS: $\mathrm{AHA}=$ American Heart Association; $\mathrm{CE}=$ contrast-enhanced; $\mathrm{Cl}=$ confidence interval; $\mathrm{HR}=$ hazard ratio; MDCTA = multidetector row $\mathrm{CT}$ angiography; $\mathrm{MRDTI}=\mathrm{MR}$ direct thrombus imaging; OCT = optical coherence tomography; TOF = time-of-flight

S troke is a leading cause of death and the major cause of long-term disability worldwide. ${ }^{1-4}$ Approximately $80 \%$ of strokes are ischemic and are thought to occur through embolic events or stenosis of cerebral arteries. More specifically, 20\%$30 \%$ of cerebral infarction has been correlated to carotid atherosclerotic plaque and artery stenosis. ${ }^{5,6}$ Morphologically, features such as carotid artery intima-media thickness, ${ }^{7-9}$ rupture-prone plaques with thin fibrous caps and lipid cores, ${ }^{10-12}$ and ulcerated plaques ${ }^{13,14}$ have all been correlated with an increased risk for stroke. Such high-risk features may be present even in patients with mild or moderate degrees of carotid stenosis. Conversely, plaques with high superficial calcium content have been associated with a lower risk for stroke. ${ }^{15}$ Noninvasive imaging techniques have been developed to extract these carotid plaque features by using sonography, ${ }^{16-18}$ MR imaging, ${ }^{19-22}$ and CT. ${ }^{14,19,23,24}$ The characterization of carotid artery plaque presents an opportunity to quantify a patient's risk of cerebrovascular events and may be used to improve the therapeutic decision-making process, such as carotid endarterectomy or angioplasty and stent placement. We present the current state-of-the-art, reflect on future highresolution quantitative vulnerable carotid plaque imaging techniques with discussions of their potential as predictors of the occurrence of cerebrovascular accidents, and refine our understanding of stroke risk stratification.

From the Biophotonics and Bioengineering Laboratory (B.A.S., V.X.D.Y.), Department of Electrical and Computer Engineering, and Medical Physics Program (V.X.D.Y.), Department of Physics, Ryerson University, Toronto, Ontario, Canada; Division of Neurosurgery (J.S., V.X.D.Y.), University of Toronto, Toronto, Ontario, Canada; Department of Medical Imaging (J.S., T.R.M., W.M.), St. Michael's Hospital, Toronto, Ontario, Canada; and Imaging Research (V.X.D.Y.), Sunnybrook Health Sciences Center, Toronto, Ontario, Canada.

Please address correspondence to Victor X.D. Yang, MD, Ryerson University, 350 Victoria St, Toronto, Ontario, Canada, M5B 2K3; e-mail: yangv@ee.ryerson.ca

Indicates open access to non-subscribers at www.ajnr.org

http://dx.doi.org/10.3174/ajnr.A2753

\section{Atherosclerotic Plaque Identification}

Atherosclerosis is characterized by progressive thickening of the arterial wall, with the deposition of cholesterol, inflammatory cell infiltration, extracellular-matrix formation, and thrombosis. ${ }^{25}$ A large portion of the general population exhibits nonsymptomatic atherosclerosis; in late-stage disease, symptoms become apparent and are usually caused by arterial stenosis and/or embolic events. Progressive atherosclerotic plaque formation can limit blood flow to key organs, such as the heart or brain, impairing function, while acute or sudden plaque rupture and subsequent thrombosis can cause myocardial infarction or stroke.

Traditional diagnosis and classification of atherosclerosis was possible only in late-stage disease formation, where arterial occlusion or stenosis were revealed through angiography or organ perfusion measurements. ${ }^{26,27}$ Due to recent advancements in medical imaging, new opportunities exist to explore and assess both the arterial morphology of blood vessels and the actual stage of atherosclerosis formation within the lumen wall itself by using higher resolution modalities. Pathology is the current criterion standard of atherosclerosis classification (Table 1). Therefore to critically evaluate the ability of these new medical imaging modalities to quantify and possibly predict ischemic or hemorrhagic stroke, one must determine which characteristics of lesion formation will result in evidence-based improved cerebrovascular care.

From Table 1, several characteristics become apparent as key metrics for the identification and staging of atherosclerotic plaque progression. Specifically, at the cellular level, if the presence and distribution of macrophages and/or foam cells could be detected, early lesion formation could be identified and tracked with time to determine the progression of cardiovascular disease. However, one could argue that because type I/II lesions are present in almost the entire general population, this identification may provide limited insight into its ability to predict the occurrence of a stroke. Additional markers that 


\begin{tabular}{|c|c|}
\hline $\begin{array}{l}\text { Histologic Classification of } \\
\text { Atherosclerosis }\end{array}$ & Characteristics \\
\hline \multicolumn{2}{|l|}{ Initial lesions } \\
\hline Type I & $\begin{array}{l}\text { Increase in the number of macrophages and appearance of foam cells distributed at random, present in } 45 \% \text { of } \\
\text { children younger than } 8 \text { months of age }\end{array}$ \\
\hline Type II (fatty streak) & $\begin{array}{l}\text { First visible lesion; layers of foam cells and drops of fat inside the smooth muscle cells and in the extracellular } \\
\text { space; minute lipid particles with round and heterogenous aspect, present in } 65 \% \text { of teenagers }\end{array}$ \\
\hline Type Ila & Intimal thickening, lesions tending to progress \\
\hline Type Ilb & Intimal tunica intima thinner and few smooth muscle cells \\
\hline \multicolumn{2}{|l|}{ Intermediary lesions } \\
\hline Type III & Lesions similar to those of type II, with extracellular lipid collections \\
\hline \multicolumn{2}{|l|}{ Advanced lesions } \\
\hline Type IV (atheroma) & $\begin{array}{l}\text { Confluence of lipid collections of type II lesions creates an extracellular dense accumulation of fat in a well- } \\
\text { defined area of the tunica intima, the lipid nucleus; no marked fibrous tissue or presence of complications } \\
\text { such as flaws on the surface of the plaque or thrombosis; between lipid nucleus and endothelial surface, } \\
\text { there is the extracellular matrix rich in proteoglycans and cells, such as lymphocytes, macrophages, and } \\
\text { foam cells; these are not usually associated with the lumen stricture of the vessel; on the contrary, there } \\
\text { may be an increase in diameter when measured from the adventitia }\end{array}$ \\
\hline Type V & $\begin{array}{l}\text { When fibrous capsule develops, it usually causes lumen stricture and can have fissures, hematomas, or } \\
\text { thrombosis }\end{array}$ \\
\hline Type Va (fibroatheroma) & $\begin{array}{l}\text { Fibrous tissue containing lipid nucleus; new lipid nuclei can appear in different locations and planes, creating } \\
\text { asymmetric lesion }\end{array}$ \\
\hline Type Vb & Calcification of fibrous nucleus or of other parts of lesion \\
\hline Type Vc & Absence or minimal presence of lipid nucleus \\
\hline \multicolumn{2}{|l|}{ Complicated lesions } \\
\hline Type VI & Usually type IV or V lesions presenting rupture on surface, hematoma or hemorrhage, and also thrombus. \\
\hline Type Vla & Surface rupture \\
\hline Type VIb & Hematoma or hemorrhage \\
\hline Type Vlc & Thrombosis \\
\hline Type Vlabc & Rupture, hemorrhage, and thrombosis \\
\hline
\end{tabular}

a Stary et al. ${ }^{28}$

carry a significant amount of importance include the composition, physical size, and shape of the vessel wall. This presents a challenge for all currently available imaging modalities, in that a potential solution must be capable of detecting submillimeter abnormalities, provide wide-field scanning ability, and also limit the associated cost burden required to image large populations.

\section{CT}

Previously, DSA had proved useful for the evaluation of the atherosclerotic disease state because the detection of angiographic ulceration and abnormalities are strong candidates for the prediction of plaque rupture. DSA was originally used for the detection of carotid stenosis and ulceration, ${ }^{29}$ but recent advances in CTA have supported the opinion that CTA is superior in the detection of carotid dysfunctions, such as plaque irregularities and ulcerations. Saba et $\mathrm{al}^{30}$ recently reported the specificity and sensitivity in the detection of ulcerations via MDCTA to be $99 \%$ and $94 \%$, respectively.

de Weert et $\mathrm{al}^{23}$ have taken the detection of plaque ulceration 1 step further in a prospective study that was composed of patients with ischemic cerebrovascular disease in which the plaque surface morphology, severity of stenosis, and cardiovascular risk factors were related to the type of cerebrovascular symptoms. Four hundred six consecutive patients with ischemic cerebrovascular disease (ie, transient ischemic attack, amaurosis fugax, stroke) were first evaluated for the presence of atherosclerotic plaque, which subsequently was classified as smooth, irregular, or ulcerated (Fig 1). It was observed that irregular and ulcerated plaques were significantly different
$(P<.001)$ between the lowest degree of stenosis $(0 \%-29 \%)$ and the higher degrees of stenosis $(30 \%-99 \%)$. Therefore complex plaques were more frequent in a higher degree of carotid artery stenosis. Although promising, their results also demonstrated limitations with this approach because atherosclerotic plaques were found in both symptomatic (55\%) and asymptomatic $(56 \%)$ carotid arteries with a slightly higher presence of complex plaques in symptomatic patients $(25 \%$ versus $18 \%$, respectively). If MDCTA is to be used as a predictor of acute stroke, additional CTA imaging metrics are required to separate high-risk patients so that they receive appropriate medical attention.

Several authors speculated that atherosclerotic lesions which contain a large necrotic core that is separated from the lumen by a fissured fibrous cap represent an increased stroke risk. ${ }^{31,32}$ Saba and Mallarini ${ }^{24}$ presented a retrospective imaging review $(n=147)$ of an MDCTA technique to evaluate the association between the presence of a fissured fibrous cap and ipsilateral symptoms. They used 4 imaging criteria to define the fissured fibrous cap; all 4 criteria needed to be present to confirm the formation of a fissured fibrous cap (Fig 2). Their results indicated a weak trend in the observation of more frequent fissured fibrous caps when the carotid artery exhibited a higher degree of stenosis: ${ }^{33} 11.76 \%$ in class IV stenosis, $16.92 \%$ in class Va stenosis, and $20 \%$ in Class Vb stenosis. Of the 147 patients, 15 were excluded due to poor MDCTA image quality. Of the remaining 132 patients, in 36 symptomatic patients, $12(33.3 \%)$ ipsilateral fissured fibrous caps were detected; and in the 96 asymptomatic patients, 11 (11.5\%) fissured fibrous caps were detected. This finding resulted in the 


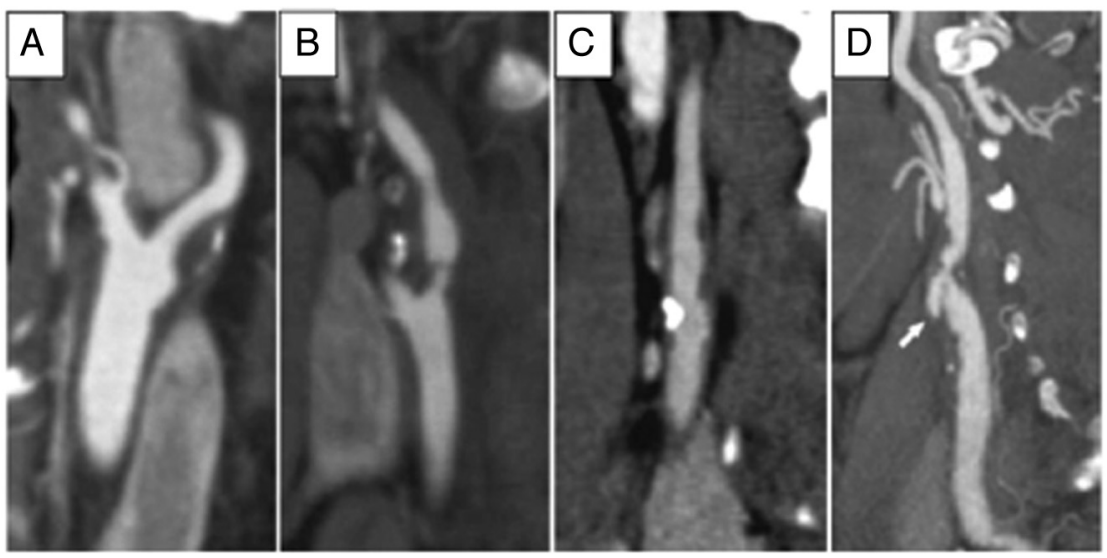

Fig 1. Multiplanar reformat images (1 mm thick). $A$, Smooth atherosclerotic carotid plaque surface. $B$ and $C$, Irregular plaque surface. $D$, Atherosclerotic carotid plaque ulceration. Arrow in $D$ demonstrates extension of contrast material beyond the vascular lumen into the surrounding plaque. Reproduced with permission from de Weert et al. Stroke 2009;40:1334-40. ${ }^{23}$

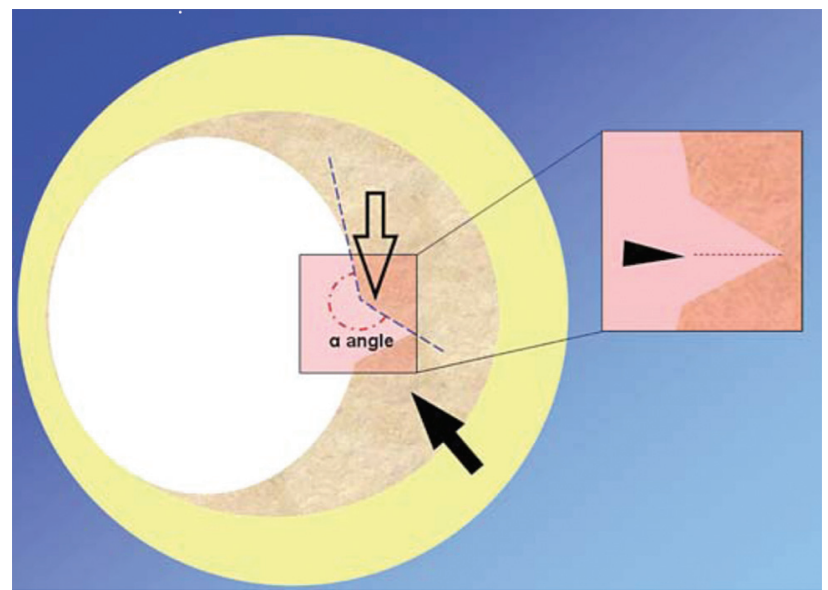

Fig 2. Representation of a carotid artery with a fissured fibrous cap. The 4 imaging criteria are depicted as follows: 1) presence of an "in-plus" image opening into a fibrous cap (open arrow); 2) a depth of $\leq 1 \mathrm{~mm}$ (arrowhead); 3 ) the angle of $\geq 230^{\circ}$ with respect to the lumen; and 4) the presence of atherosclerotic plaque into which the "in-plus" image projects (arrow). Reproduced with permission from Saba and Mallarini. Cerebrovasc Dis 2009;27: $322-27^{24}$ and S. Karger, AG, Basel.

presence of a statistically significant $(P=.003)$ association between the detection of a fissured fibrous cap and ipsilateral symptoms. The authors concluded with the observation that fine surface features of atherosclerotic plaques could play an important role in risk stratification and merit additional investigation.

Because the progression of atherosclerosis is a complex biologic event, additional imaging metrics are required to fully identify a patient's risk for stroke, based not only on luminal surface features but also on subsurface tissue morphology. Building on their previous MDCTA studies, which quantitatively identified structures such as carotid plaque calcification, large lipid cores, detection of ulcerations, and fissured fibrous cap thickness measurements, Wintermark et al ${ }^{14}$ subsequently used these imaging metrics to retrospectively investigate their association with the occurrence of ischemic stroke. ${ }^{34}$ The study consisted of a consecutive series of 136 patients, of whom 40 patients with carotid stroke and 50 patients without carotid stroke were considered for MDCTA image analysis, after patient review. The image postprocessing consisted of an automated classifier computer algorithm that had been previ-

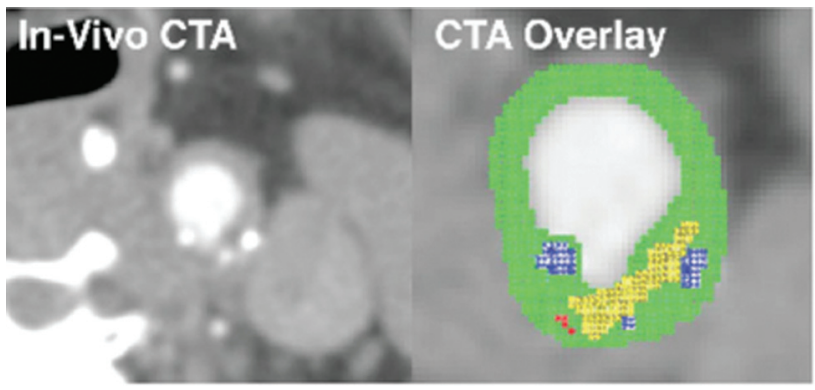

Fig 3. In vivo CTA image of the common carotid artery and automated classification computer algorithm-derived overlay highlighting the lipid-rich necrotic core in yellow, calcification in blue, blood products in red, and the remaining connective tissue in green. Reproduced with permission from Wintermark et al. AJNR Am J Neuroradiol 2008:29:875-82. ${ }^{14}$

ously validated, which extracted features of the $3 \mathrm{~cm}$ on each side of the carotid bifurcation. ${ }^{14}$ The algorithm segmented the inner and outer contours of the carotid artery wall and distinguished different components, such as lipids and calcium. These are displayed in a color overlay on the CT dataset for ease of visualization (Fig 3). The algorithm then quantifies, in $3 \mathrm{D}$, several features such as lumen area, carotid wall volume, number of calcium clusters, number of lipid clusters, fibrous cap thickness, and so forth. This technique demonstrated that a small number of carotid wall MDCTA features (ie, increased wall volume, greater number of lipid clusters, and lipid clusters that were closer to the lumen) were significantly associated with patients with acute carotid stroke.

CT has demonstrated its clinical utility for accurate characterization of the degree of carotid stenosis but has limited carotid wall characterization outside of calcium content as a predictor of stroke. Advantages of MDCTA are that it is part of the standard of care for patients admitted with cerebrovascular disease, is widely available within hospital infrastructure, and with additional multicenter validation, presents an opportunity to improve stroke risk stratification and provide new perspectives for preventative treatment.

\section{MR Imaging}

It is generally accepted that plaque rupture and subsequent luminal thrombosis formation may be the most important mechanisms leading to acute ischemic stroke. ${ }^{31}$ High-resolu- 


\begin{tabular}{ll}
\hline $\begin{array}{l}\text { Table 2: Conventional AHA classification of atherosclerotic plaques } \\
\text { adapted for MR imaging }\end{array}$ \\
\begin{tabular}{ll}
\hline \multicolumn{1}{l}{ Type of lesion } & \multicolumn{1}{c}{ Characteristics } \\
\hline $\begin{array}{l}\text { Type I-II } \\
\text { Type III }\end{array}$ & $\begin{array}{l}\text { Thin plaque }(<10 \% \text { stenosis), with no calcification } \\
\text { Type IV-Va }\end{array}$ \\
Pype Vb & $\begin{array}{l}\text { Plaque with small lipid cores and no calcification } \\
\text { fibrous cap, possible small calcifications } \\
\text { Plaque with a lipid core or fibrotic tissue, with } \\
\text { large calcifications } \\
\text { Type Vc }\end{array}$ \\
Type Vlb-Vlc & Plaque with fibrous tissue, no lipid core, possible \\
small calcifications
\end{tabular} \\
\hline
\end{tabular}

tion MR imaging has become an integral component for the evaluation of transient ischemic attacks and strokes and seems to be particularly useful for investigating the morphology of the carotid artery. Specific examples include the ability to discriminate large lipid cores with macrophage infiltration, thin and fragile fibrous caps, calcified nodules, characterization of intraplaque hemorrhage, and acute thrombosis. ${ }^{35,36}$ Serfaty et $\mathrm{al}^{37}$ have proposed and published an adapted AHA classification of atherosclerotic plaque specifically for MR imaging. This classification (Table 2) highlights the ability of MR imaging to organize the plaque on the basis of composition and morphology, specifically the detection of small and large lipid core differences and the presence of calcifications and plaques with hemorrhage or thrombosis.

Various MR imaging signal-intensity acquisition techniques have been investigated to optimize the detection of the lumen wall in carotid arteries in combination with bolus injections for contrast enhancement. 3D TOF MR angiographic acquisition with thin sections, small voxels, and short TEs has been used to achieve higher resolution images. However, this technique may still have signal-intensity loss due to unpredictable transit time, which can be particularly troublesome in stenotic arterial segments, where the residual lumen can be very small; the size makes accurate lumen border detection and composition quite challenging. ${ }^{38}$ An alternative approach to quantify stenotic regions consists of a time-resolved $3 \mathrm{D}$ acquisition technique that repeatedly images a vessel of interest during the passage of a bolus of contrast agent. This technique has been termed time-resolved imaging of contrast kinetics and provides $3 \mathrm{D}$ datasets with a temporal resolution of 2-6 seconds, yielding an angiographic series similar to standard angiography. ${ }^{39,40}$ Therefore, this technique can identify vessels that may opacify late in the imaging sequence, which may prove to be advantageous over non-time-resolved contrast-enhanced techniques because the contrast enhancement usually provides a single snapshot during the peak of contrastbolus perfusion. However, diseased or stenotic vessels with slow flow rates may opacify late; therefore, these important vessels (or vessel defects) may still not be optimally visualized.

Although MR imaging is generally well-suited for imaging the soft-tissue components of carotid vulnerable plaques, can these measurements be used to predict subsequent stroke? Gao et $\mathrm{al}^{22}$ have recently released a study in which high-resolution CE-MRI was compared with subsequent histologic sections from specimens to assess the accuracy of MR imaging (Fig 4), followed by an evaluation of the relationship between the carotid plaque characteristics and their various types of associated strokes.

Their single-center prospective study consisted of 102 consecutive patients, in which the patients were assigned to 4 different categories according to the degree of carotid artery stenosis as measured by sonography: 50\%, 51\%-69\%, 70\%$89 \%$, and $90 \%-100 \%$. The patient population was then further classified into 4 groups on the basis of MR imaging atherosclerotic lesion observations; type III, invulnerable plaques; types IV $-\mathrm{V}$, lipid/necrotic core, fibrous cap, possible calcification; type VI, surface defect and intraplaque hemorrhage; and type VII, calcified plaques. Their findings demonstrated that 45 patients had carotid stroke and 55 patients (2 patients were removed from study due to poor image quality) had lacunar stroke and asymptomatic infarction. They observed a significant correlation $(P<.001)$ between the presence of mild-to-moderate stenosis $(\leq 70 \%)$ and types IV $-\mathrm{V}$ vulnerable plaque. In patients with carotid stroke $(n=45)$, 30 had thin or ruptured fibrous caps, twice the number that exhibited thick and intact fibrous caps. These results were consistent with previous literature and indicate that high-resolution CE-MRI may provide useful information for the appropriate selection of candidates for invasive treatment and that the identification of morphologic mechanisms may, in fact, be predictive of stroke.

Takaya et al $^{12}$ also used MR imaging as a powerful tool to quantify carotid plaque on the basis of the plaque characteristics as a predictive measure of future ipsilateral cerebrovascular events. ${ }^{12}$ One hundred fifty-four consecutive patients who had asymptomatic 50\%-79\% carotid stenosis (measured by sonography) were scanned by MR imaging at the beginning and every 3 months to a mean follow-up time of 38.2 months to identify symptoms of cerebrovascular events. Significant associations between the baseline MR imaging and subsequent symptoms included the presence of a thin or ruptured cap resulting in an HR of $17.0(P \leq .001)$, and the occurrence of intraplaque hemorrhage producing an HR of $5.2(P=.005)$. These results demonstrated the potential usefulness of MR imaging to provide predictive measurements to assess patient risk and form the bases for large multicenter studies to confirm these results.

Murphy et $\mathrm{al}^{41}$ have shown that MR imaging has the ability to discriminate between different stages of thrombus and hemorrhage formation, via the formation of methemoglobin during the acute/subacute phase, resulting in the signal-intensity increase of the T1WI sequence. This technique was used for in vivo detection of carotid plaque in the ipsilateral arteries of symptomatic patients with suspected stenosis of the carotid artery. These findings were then compared with the authors' own contralateral arteries and with those of healthy sex- and age-matched controls. ${ }^{42}$ An MRDTI scan with positive findings was diagnosed if there was high-signal-intensity content within the wall or lumen of the carotid artery in the proximity of the lesion and $1 \mathrm{~cm}$ on either side of the stenosis. This observation (or lack of) was recorded for both the ipsilateral (symptomatic) and the contralateral (asymptomatic) artery for each patient; a sample MRDTI image can be seen in Fig 5. A total of 134 patients were imaged by using MRDTI, consisting of patients $(n=120)$ with suspected severe carotid artery stenosis (70\%-100\%) and previous acute cerebral ischemia; 

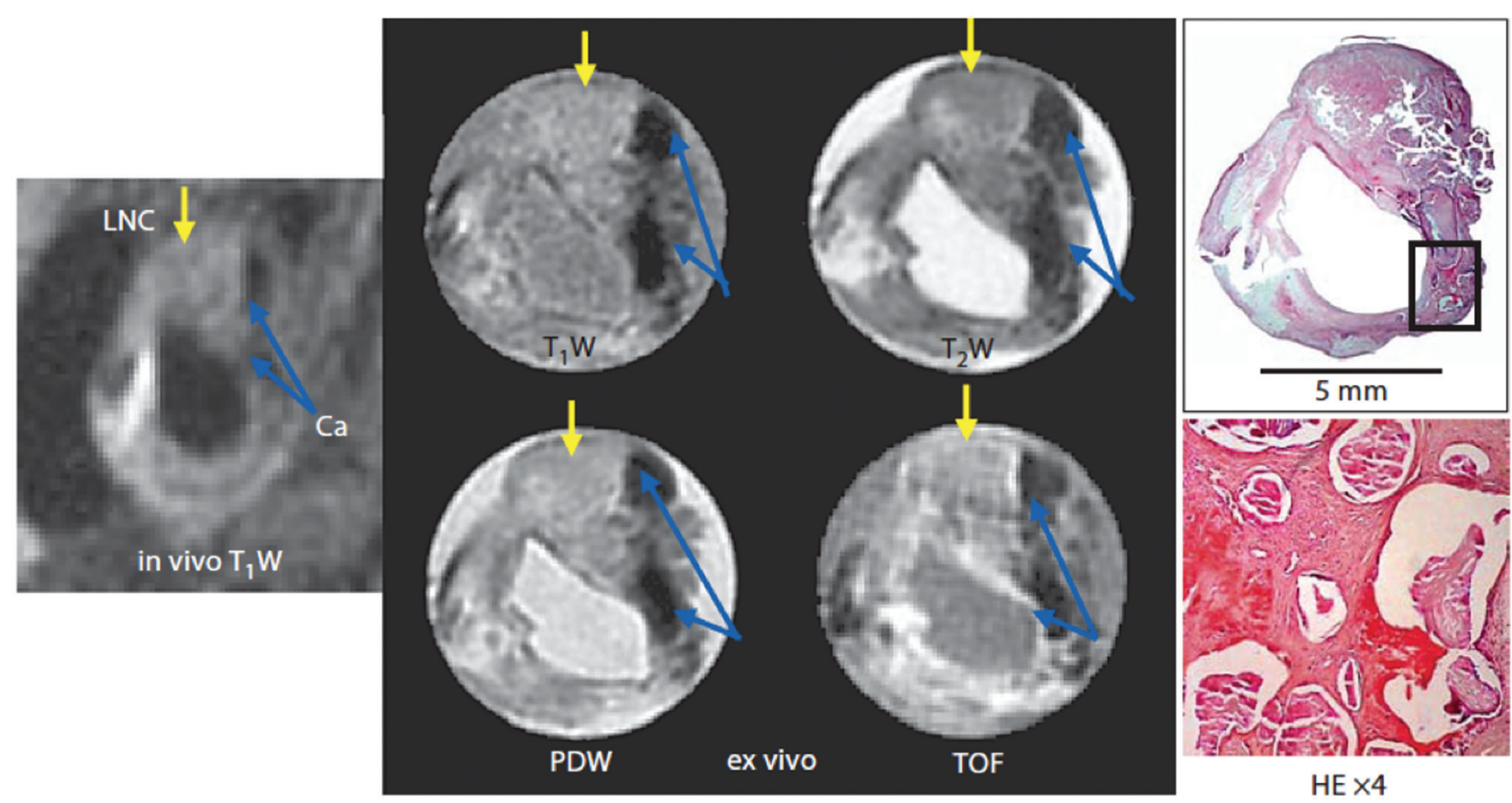

Fig 4. Carotid artery bifurcation vulnerable plaque of in vivo and ex vivo samples as seen on MR imaging (T1W, TOF, proton density-weighted [PDW], and T2-W) along with the corresponding matched H\&E histologic cross-section. The external carotid artery is seen just above and to the right of the internal carotid artery. The external carotid artery was occluded with a large lipid necrotic core (LNC). Areas of dense calcification (Ca) appear as a dark region from the 1- to 5-o'clock position on MR imaging and the histologic cross-section of the internal carotid artery. The H\&E-stained histology section confirms the presence of a superficial calcified nodule. Reproduced with permission from Gao et al. Cerebrovasc Dis 2009;27:345-5222 and S. Karger, AG, Basel.
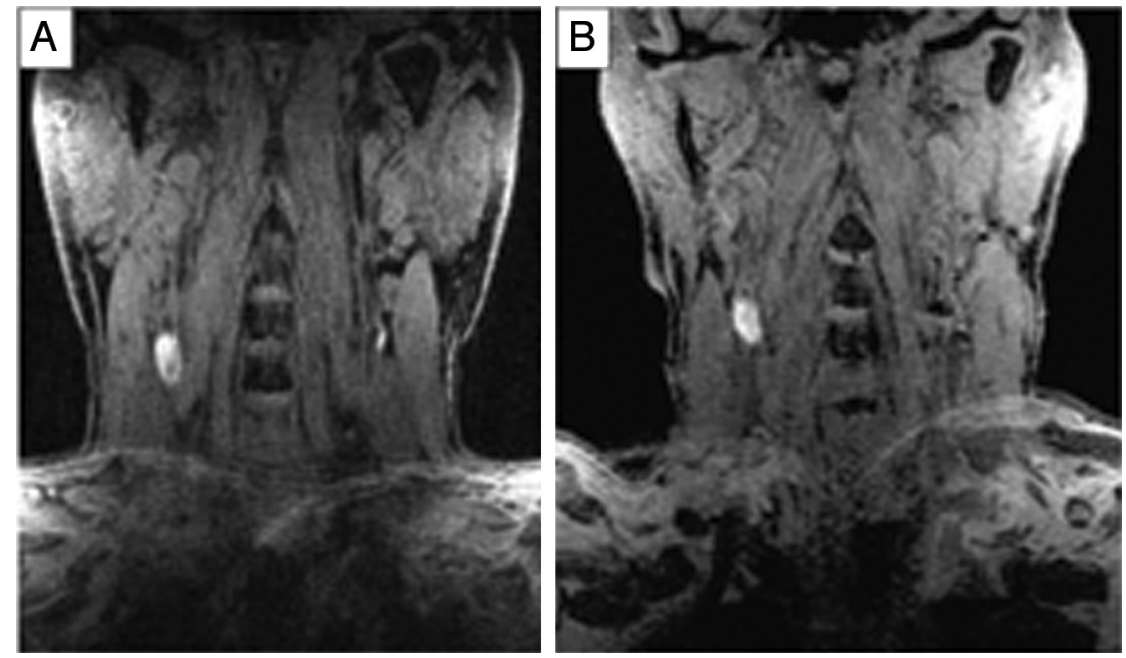

Fig 5. $A$, High-signal-intensity material within the right internal carotid artery (symptomatic side). Note a smaller volume of asymptomatic complicated plaque on the left. $B$, Unilateral right internal carotid disease, whereas no observable high signal intensity is seen on the left (asymptomatic) side. Reproduced with permission from Murphy et al. Circulation 2003;107:3053-58. ${ }^{41}$

28 control arteries $(n=14)$ were also imaged. The control subjects presented with no observable high signal intensity in their arteries, while there was a $60 \%$ occurrence of high signal intensity in the patients' ipsilateral arteries, suggesting the presence of complicated plaques. This observation was significantly greater in the patients' ipsilateral carotid artery, compared with the contralateral asymptomatic side at $60 \%$ versus $36 \%\left(\chi^{2}, P<.001\right)$, respectively.

These MR imaging results suggest that the various methods of obtaining carotid plaque contrast may play a potential role in risk stratification, in which the development of appropriate management strategies, based on the identification of prob- lematic plaques, may lead to an improvement in overall patient mortality and morbidity. Some of the hurdles faced by such a carotid MR imaging technology are the cost, availability, and length of imaging time.

\section{Sonography}

Doppler sonography offers an inexpensive, noninvasive, portable, and clinically accepted solution for imaging the carotid artery to assess the severity of stenosis, an established risk factor for stroke, ${ }^{43}$ by quantifying peak systolic and peak diastolic velocity and/or their ratio. ${ }^{16} \mathrm{~B}$-mode sonography of the carotid intima-media thickness has also recently become a noted 


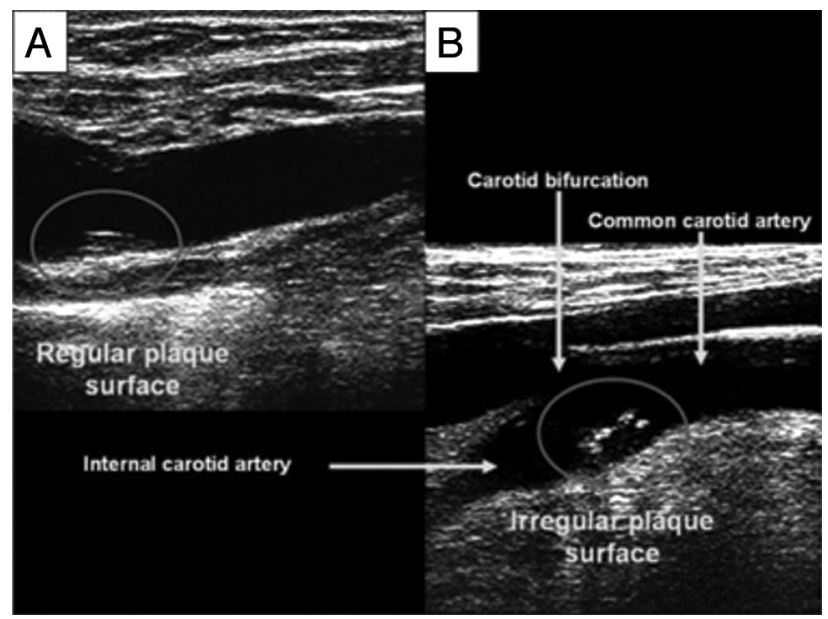

Fig 6. $A$, Regular carotid plaque surface. $B$, Irregular carotid plaque surface. Reproduced with permission from Prabhakaran et al. Stroke 2006;37:2696-701. ${ }^{13}$

characteristic for assessment of cardiovascular risk. ${ }^{44,45}$ Stateof-the-art 3D sonographic systems can now also provide measurement of the total plaque volume and area ${ }^{46-48}$ and lumen wall volume ${ }^{41}$; preliminary (ex vivo) research has validated the ability of sonography to identify different carotid plaque components. ${ }^{50}$

Rundek et $\mathrm{al}^{51}$ have recently reported an association between the maximum carotid plaque thickness and the risk of cerebrovascular events. Carotid plaque imaging, via high-resolution B-mode sonography in 2189 subjects, was analyzed; patients were separated into 3 groups: 1) no plaque, 2) plaque with $<1.9 \mathrm{~mm}$ thickness (value associated with the 75 th percentile of the maximum carotid plaque thickness distribution), and 3 ) $\geq 1.9-\mathrm{mm}$ carotid plaque thickness. The primary end point of the study was the occurrence of a vascular event such as ischemic stroke, myocardial infarction, or vascular death. Carotid plaque was present in 1263 (58\%) subjects, and after a mean follow-up period of 6.9 years, a vascular event occurred in 319 subjects, of whom 121 experienced a fatal or nonfatal ischemic stroke. The authors concluded that maximum carotid plaque thickness is a simple marker of subclinical atherosclerosis; patients with $\geq 1.9-\mathrm{mm}$ carotid plaque thickness had a 2.8-fold increase in the risk of ischemic stroke (HR, 2.80; 95\% CI, 2.04-3.84).

A large prospective study carried out by the Stroke and Critical Care Division of Columbia University recently evaluated the association of carotid plaque surface irregularities and the associated risk of ischemic stroke in patients. ${ }^{44}$ High-resolution (13 MHz) B-mode sonography was performed in 1939 stroke-free subjects, in whom the presence of carotid plaque was defined as a focal protrusion of $>50 \%$ of that of the surrounding area and localized to the internal carotid artery bifurcation. The plaque surface was then identified as either regular or irregular (Fig 6), and the subjects were followed for a mean period of 6.2 years, during which time 69 ischemic strokes occurred. It was discovered that there was nearly a 3 -fold increase ( $8.5 \%$ versus $3.0 \%$ ) for ischemic stroke in subjects exhibiting an irregular plaque surface compared with a regular plaque surface. When adjusted for demographics, traditional risk factors, the degree of plaque thickness, and the presence of an irregular plaque were independently associated with ischemic stroke versus the presence of no plaque (HR, 3.1; 95\% CI, 1.1-8.5). Therefore, plaque surface irregularities detected by sonography may serve as a useful and easily attainable marker in the high-risk patient. It remains to be seen how sonographic operator dependencies may affect the reproducibility of the plaque irregularity metric.

With further clinical investigation, sonography may play an important role as a cost-effective, easy-to operate primary risk-assessment tool, which may appropriately stratify patients for more cost-intensive or invasive procedures, thus improving overall patient care. The development of advanced 3D sonographic techniques may reduce the operator dependency, which has been a traditional drawback of this technique.

\section{Current Imaging Developments: Endovascular OCT}

According to the American Heart Association classification of atherosclerotic lesions (Table 1), important markers of vessel wall disease progression include the presence of macrophages, foam cells, and intimal thickening and clear identification of fissures. ${ }^{28}$ Although the previously mentioned imaging modalities of CT, MR imaging, and sonography can identify key markers of atherosclerosis, these minute cellular markers are below their respective imaging-resolution capabilities. One potential real-time imaging solution to identify these structures is OCT. ${ }^{52}$ OCT is an imaging technique that can visualize subsurface tissue architecture in highly resolved detail $(\sim 10$ $\mu \mathrm{m}$ in tissue). Although, to our knowledge, in vivo OCT carotid artery imaging studies do not currently exist or have not been reported, this technique has had clinical success in the characterization of the coronary artery. To date, OCT characteristics such as lipid content, fibrous cap thickness, and fibrous cap macrophage attenuation have been validated by using histologic controls. ${ }^{53-56}$

The high spatial resolution of OCT may be an ideal in vivo imaging technology to assess the thickness of the fibrous tissues overlying an arterial plaque. Clinical results have demonstrated that a thin-cap fibroatheroma of $<65 \mu \mathrm{m}$ has a high correlation with the probability of a plaque rupture in the coronary artery. ${ }^{57}$ Therefore, when designing or implementing a vessel wall imaging standard, the resolution of the imaging system becomes a key component to identify areas of potential plaque rupture. OCT has been shown to clearly identify these key morphologic features, including not only thin-cap fibroatheroma but also the different arterial vessel wall layers, including the intimal layer, the media, and the outer layer. Additionally, the characterization of the different types of plaque elements such as fibrous, calcific, and lipid rich ones and the presence and infiltration of macrophages have been accomplished. ${ }^{53}$ The macrophage attenuation may be a particularly useful in vivo measurement because it provides a measure of the inflammation within a plaque region. ${ }^{55} \mathrm{Al}-$ though the process of gaining access to the carotid or coronary arties requires a minimally invasive access point, such as the femoral artery or carotid artery, the fiber-optic delivery of the imaging beam is highly amenable to miniaturization. This allows the use of side-viewing probes ( $\sim 300 \mu \mathrm{m}$ in diameter $)$ to be used in an attempt to reduce the invasiveness and complexity of the imaging procedure. ${ }^{58}$

Because OCT is the most recent imaging technique to enter the vascular imaging arena, some may view this technique as 

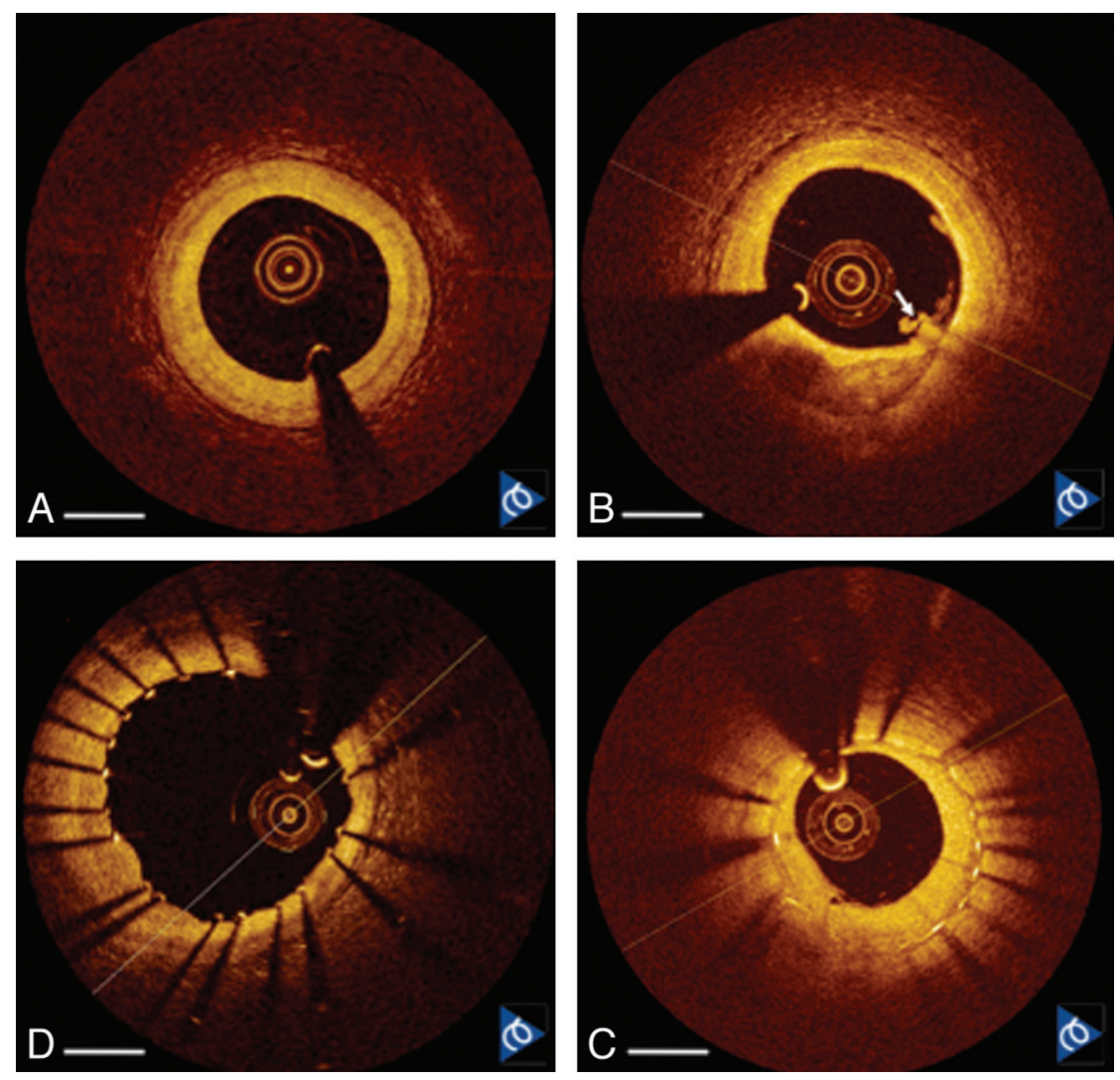

Fig 7. Frequency-domain OCT images of in vivo human coronary arteries acquired at 50,000 image lines per second. $A$, Normal artery with mild intimal hyperplasia. $B$, Calcium plaque $(6$ o'clock) with a small thrombus (arrow). C. Freshly implanted stent showing good apposition. D, Previously implanted stent at follow-up showing neointimal thickening and restenosis. Scale bar $=1 \mathrm{~mm}$. Image courtesy of LightLab.

too premature for direct comparison with the previously mentioned techniques (ie, CT, MR imaging, and sonography) and its potential use in carotid artery imaging. Evolution to carotid imaging may come from critically analyzing the recent introduction of a US Food and Drug Administration-approved (C7-XR; LightLab, Westford, Massachusetts) intravascular OCT imaging system for cardiology. This system has been used in vivo to image the normal coronary artery (with or without mild intimal thickening), coronary atherosclerotic plaques (with or without calcification), and thrombosis or dissection pathologies. Pre- and poststenting OCT allowed evaluation of stent placement, neointimal hyperplasia, and restenosis, as shown in Fig 7.

As the OCT technology matures, this technology may become a standard tool to image the coronary artery as a means of detecting and stratifying the progression of atherosclerotic plaque. This has laid the groundwork for a detailed assessment of heart disease and may also be vital in developing an imaging tool to detect and quantify the risk for stroke through investigation of the atherosclerotic plaque in the carotid artery.

\section{Future Speculation and Overall Conclusions}

Several noninvasive imaging techniques such as MR imaging, $\mathrm{CT}$, and sonography have shown promise in detecting the fine surface and subsurface morphology of atherosclerotic plaques in the carotid arterial wall. However, these techniques could benefit from higher resolution to image morphologic signatures associated with plaque rupture, including intima-media thickness, macrophage infiltration, and the presence and thickness of a thin-cap fibroatheroma. When these histologically observed markers are present in the carotid artery, they have been correlated with an increased risk for stroke leading to mortality or morbidity. Therefore, should one ask whether the benefits of a high-resolution invasive imaging technique outweigh the potential complications of endovascular carotid imaging?

If we look to previous research concerning the use of a high-resolution system (ie, high-frequency sonography or OCT), specifically in coronary artery imaging, as a preliminary model for success to lay the foundation and extension into carotid artery imaging, one needs to understand and investigate how this imaging scenario could be envisioned. In coronary artery imaging, the proper deployment of a stent is extremely important because the cardiologist uses a balloon-expandable stent in the coronary artery to maintain a minimum luminal area. However, the interventional neuroradiologist uses a self-expanding stent in the carotid artery. Perhaps a more important use of a high-resolution endovascular imaging system may include the characterization of atherosclerotic plaque and how its morphology relates to symptomatic or nonsymptomatic patients with stroke. Cardiologists must also pay attention to the presence and size of any intimal hyperplasia in the coronary artery, whereas due to the much larger diameter of the carotid artery, a similar amount of hyperplasia usually results in limited clinical significance; therefore, we believe this useful coronary imaging metric may be less useful in carotid evaluation. Intracranial stent placement for lesions 
such as giant aneurysms using the new generation of flowdiversion devices, ${ }^{59}$ in which the parent vessel diameter is smaller than the proximal internal carotid artery, may benefit from such detailed imaging of neointimal hyperplasia.

At this stage of interventional carotid imaging, we believe the most potentially beneficial implication of an in vivo highresolution imaging technique would be to characterize carotid plaques before stent placement and to also image poststent placement in an effort to identify any stent-plaque beneficial or detrimental interactions. An initial pre-/post-stent placement prospective study could provide similar quantifiable imaging metrics, such as the thickness of the fibrous cap in the coronary artery, as an assessment of stroke risk.

An additional important point to consider when comparing coronary and carotid artery imaging lies with the practicality of imaging and the intervention in these different vessels. In the past several decades, many new intravascular technologies (eg, intravascular sonography, OCT, and so forth) have been incorporated into the routine care for patients with coronary disease undergoing cardiac catheterization procedures. This is in stark contrast to the carotid artery, in which, for the past several years, there has been an effort to avoid invasive procedures for diagnostic purposes. Therefore, the risk assessment of implementing carotid wall imaging must demonstrate that the benefit of in vivo high-resolution imaging, which currently lies outside the resolution capabilities of existing technologies, including multisection CT, MR imaging, and sonography, must outweigh the risk of invasive diagnostic angiography. Madjid et $\mathrm{al}^{60}$ point out that though the usefulness of locating vulnerable plaque is unproved, prognosis is very valuable to the patient. For example, a patient may postpone travel arrangements or choose elective surgery to address the identified problems. Proper analysis of the risk trade-off from extrapolated data of previous clinical trials and adequate design of new prospective studies remain the key to discussions for evaluating the potential of carotid endovascular imaging.

We believe that a stepwise plan can be designed for highresolution endovascular imaging, (ie, high-frequency sonography or OCT), during carotid angioplasty and stent placement, in which the primary goal will be immediate assessment of carotid plaque and vessel stenosis pre- and post-balloon angioplasty. A secondary goal would be in vivo characterization of the carotid plaque and comparison with noninvasive imaging. A proper assessment of the risk could then be accomplished as the benefits of this invasive imaging technique are compared with the benefits of endovascular carotid imaging. With this accomplished, one could then envision using these techniques in diagnostic or prognostic applications, where the endovascular imaging method may be integrated into the existing patient management algorithm by using a multimodality approach (ie, sonography, CT, MR, OCT) to identify the vulnerable carotid lesions with high-risk profiles in the lowto-moderate stenosis categories.

Disclosures: Thomas R. Marotta-Unrelated: Consultancy: ev3, Comments: proctor for Pipeline device; Employment. private practice. Victor X.D. Yang-Related: Grant. Natural Sciences and Engineering Research Council of Canada, ${ }^{*}$ Comments: Canada research chair; Other. LightLab Imaging, ${ }^{*}$ Comments: in-kind engineering support. *Money paid to the institution.

\section{References}

1. Murray C, Lopez A. Mortality by cause for eight regions of the world: Global Burden of Disease Study. Lancet 1997;349:1269-76

2. CANSIM Table 102-0529: Deaths, by cause, Chapter IX: Diseases of the circulatory system (100 to 199), age group and sex, Canada, annual, 2000 to 2006. Statistics Canada Released May 4, 2010.

3. Lloyd-Jones D, Adams R, Carnethon M, et al. Heart disease and stroke statistics: 2009 update-a report from the American Heart Association Statistics Committee and Stroke Statistics Subcommittee. Circulation 2009;119: $480-86$

4. Allender S, Scarborough P, Peto V, et al. European cardiovascular disease statistics. Brussels. European Heart Network 2008.

5. Caplan L. Diagnosis and treatment of ischemic stroke. JAMA 1991; 266: 2413-18

6. Warlow C, Dphil C, Dennis M, et al. Stroke. Lancet 2003;362:1211-24

7. Ebrahim S, Papacosta O, Whincup P, et al. Carotid plaque, intima media thickness, cardiovascular risk factors, and prevalent cardiovascular disease in men and women: the British Regional Heart Study. Stroke 1999;30:841-50

8. O'Leary D, Polak J, Kronmal R, et al. Carotid-artery intima and media thickness as a risk factor for myocardial infarction and stroke in older adults. New Engl J Med 1999;340:14

9. Lorenz M, von Kegler S, Steinmetz H, et al. Carotid intima-media thickening indicates a higher vascular risk across a wide age range: prospective data from the Carotid Atherosclerosis Progression Study (CAPS). Stroke 2006;37:87-92

10. Polak J, Shemanski L, O'Leary D, et al. Hypoechoic plaque at US of the carotid artery: an independent risk factor for incident stroke in adults aged 65 years or older-Cardiovascular Health Study. Radiology 1998;208:649-54

11. Biasi G, Froio A, Diethrich E, et al. Carotid plaque echolucency increases the risk of stroke in carotid stenting: the Imaging in Carotid Angioplasty and Risk of Stroke (ICAROS) study. Circulation 2004;110:756-62. Epub 2004 Jul 26

12. Takaya N, Yuan C, Chu B, et al. Association between carotid plaque characteristics and subsequent ischemic cerebrovascular events: a prospective assessment with MRI-initial results. Stroke 2006;37:818-23. Epub 2006 Feb 9

13. Prabhakaran S, Rundek T, Ramas R, et al. Carotid plaque surface irregularity predicts ischemic stroke: the Northern Manhattan Study. Stroke 2006;37: 2696-701. Epub 2006 Sep 28

14. Wintermark M, Jawadi S, Rapp J, et al. High-resolution CT imaging of carotid artery atherosclerotic plaques. AJNR Am J Neuroradiol 2008;29:875-82. Epub 2008 Feb 13

15. Nandalur K, Hardie A, Raghavan P, et al. Composition of the stable carotid plaque: insights from a multidetector computed tomography study of plaque volume. Stroke 2007;38:935-40. Epub 2007 Feb 1

16. Hunink M, Polak J, Barlan M, et al. Detection and quantification of carotid artery stenosis: efficacy of various Doppler velocity parameters. AJR Am J Roentgenol 1993;160:619-25

17. Zureik M, Bureau J, Temmar M, et al. Echogenic carotid plaques are associated with aortic arterial stiffness in subjects with subclinical carotid atherosclerosis. Hypertension 2003;41:519-27. Epub 2003 Feb 10

18. Spence J. Technology insight: ultrasound measurement of carotid plaquepatient management, genetic research, and therapy evaluation. Nat Clin Pract Neurol 2006;2:611-19

19. Randoux B, Marro B, Koskas F, et al. Carotid artery stenosis: prospective comparison of CT, three-dimensional gadolinium-enhanced MR, and conventional angiography. Radiology 2001;220:179-85

20. Turski P, Korosec F, Carroll T, et al. Contrast-enhanced magnetic resonance angiography of the carotid bifurcation using the time-resolved imaging of contrast kinetics (TRICKS) technique. Top Magn Reson Imaging 2001;12: $175-81$

21. Adame I, Van der Geest R, Wasserman B, et al. Automatic segmentation and plaque characterization in atherosclerotic carotid artery MR images. MAGMA 2004;16:227-34. Epub 2004 Mar 16

22. Gao T, Zhang Z, Yu W, et al. Atherosclerotic carotid vulnerable plaque and subsequent stroke: a high-resolution MRI study. Cerebrovasc Dis 2009;27: 345-52

23. de Weert T, Cretier S, Groen H, et al. Atherosclerotic plaque surface morphology in the carotid bifurcation assessed with multidetector computed tomography angiography. Stroke 2009;40:1334-40. Epub 2009 Mar 5

24. Saba L, Mallarini G. Fissured fibrous cap of vulnerable carotid plaques and symptomaticity: are they correlated? Preliminary results by using multi-detector-row CT angiography. Cerebrovasc Dis 2009;27:322-27

25. Lusis A. Atherosclerosis: review article. Nature 2000;407:233-41

26. Rothwell P, Slattery J, Warlow C. Clinical and angiographic predictors of stroke and death from carotid endarterectomy: systematic review. BMJ 1997;315:1571-77

27. Adams H, del Zoppo G, Alberts M, et al. Guidelines for the early management of adults with ischemic stroke: A Guideline From the American Heart Association/American Stroke Association Stroke Council, Clinical Cardiology Council, Cardiovascular Radiology and Intervention Council, and the Atherosclerotic Peripheral Vascular Disease and Quality of Care Outcomes in Research Interdisciplinary Working Groups-The American Academy of 
Neurology affirms the value of this guideline as an educational tool for neurologists. Stroke 2007;38:1655-534

28. Stary $\mathrm{H}$, Chandler A, Dinsmore $\mathrm{R}$, et al. A definition of advanced types of atherosclerotic lesions and a histological classification of atherosclerosis: a report from the Committee on Vascular Lesions of the Council on Arteriosclerosis, American Heart Association. Arterioscler Thromb Vasc Biol 1995;15:1512-31

29. Streifler J, Eliasziw M, Fox A, et al. Angiographic detection of carotid plaque ulceration: comparison with surgical observations in a multicenter studyNorth American Symptomatic Carotid Endarterectomy Trial. Stroke 1994;25:1130-32

30. Saba L, Caddeo G, Sanfilippo R, et al. Efficacy and sensitivity of axial scans and different reconstruction methods in the study of the ulcerated carotid plaque using multidetector-row CT angiography: comparison with surgical results. AJNR Am J Neuroradiol 2007;28:716-23

31. Carr S, Farb A, Pearce W, et al. Atherosclerotic plaque rupture in symptomatic carotid artery stenosis. J Vasc Surg 1996;23:755-66

32. Bassiouny H, Sakaguchi Y, Mikucki S, et al. Juxtalumenal location of plaque necrosis and neoformation in symptomatic carotid stenosis. J Vasc Surg 1997;26:585-94

33. North American Symptomatic Carotid Endarterectomy Trial Collaborators. Beneficial effect of carotid endarterectomy in sympathetic patients with highgrade carotid stenosis. N Engl J Med 1991;325:445-53

34. Wintermark M, Arora S, Tong E, et al. Carotid plaque CT imaging in stroke and non-stroke patients. Ann Neurol 2008;64:149-57

35. Yuan C, Kerwin W, Ferguson M, et al. Contrast-enhanced high resolution MRI for atherosclerotic carotid artery tissue characterization. J Magn Reson Imaging 2002;15:62-67

36. Saam T, Hatsukami T, Takaya N, et al. The vulnerable, or high-risk, atherosclerotic plaque: noninvasive MR imaging for characterization and assessment. Radiology 2007;244:64-77

37. Serfaty J, Chaabane L, Tabib A, et al. Atherosclerotic plaques: classification and characterization with T2-weighted high-spatial-resolution MR imaging - an in vitro study. Radiology 2001;219:403-10

38. Dix J, Skrocki J. Evaluation of carotid stenosis by angiography: potential bias toward overestimated measurements introduced by prior interpretation of Doppler sonograms. AJNR Am J Neuroradiol 2000;21:639

39. Korosec F, Frayne R, Grist T, et al. Time-resolved contrast-enhanced 3D MR angiography. Magn Reson Med 1996;36:345-51

40. Mano $Y$, Shimizu $H$, Inoue $T$, et al. Magnetic resonance angiographic occlusion of the cervical carotid artery at 3 Tesla: its pitfalls [in Japanese]. No Shinkei Geka 2008;36:51-58

41. Murphy R, Moody A, Morgan P, et al. Prevalence of complicated carotid atheroma as detected by magnetic resonance direct thrombus imaging in patients with suspected carotid artery stenosis and previous acute cerebral ischemia. Circulation 2003;107:3053-58. Epub 2003 Jun 9

42. Moody A, Murphy R, Morgan P, et al. Characterization of complicated carotid plaque with magnetic resonance direct thrombus imaging in patients with cerebral ischemia. Circulation 2003;107:3047-52. Epub 2003 Jun 9

43. Barnett $\mathrm{H}$, Taylor D, Eliasziw M, et al. Benefit of carotid endarterectomy in patients with symptomatic moderate or severe stenosis. New Engl J Med 1998;339:1415-25

44. Cao J, Thach C, Manolio T, et al. C-reactive protein, carotid intima-media thickness, and incidence of ischemic stroke in the elderly: the Cardiovascular Health Study. Circulation 2003;108:166-70. Epub 2003 Jun 23

45. Hurst R, Ng D, Kendall C, et al. Clinical use of carotid intima-media thickness: review of the literature. J Am Soc Echocardiogr 2007;20:907-14

46. Spence J, Eliasziw M, DiCicco M, et al. Carotid plaque area: a tool for targeting and evaluating vascular preventive therapy. Stroke 2002;33:2916-22

47. Landry A, Spence J, Fenster A. Measurement of carotid plaque volume by 3-dimensional ultrasound. Stroke 2004;35:864-69. Epub 2004 Mar 11

48. Landry A, Ainsworth C, Blake C, et al. Manual planimetric measurement of carotid plaque volume using three-dimensional ultrasound imaging. Med Phys 2007;34:1496-505

49. Egger M, Chiu B, Spence J, et al. Mapping spatial and temporal changes in carotid atherosclerosis from three-dimensional ultrasound images. Ultrasound Med Biol 2008;34:64-72

50. Lal B, Hobson R, Hameed M, et al. Noninvasive identification of the unstable carotid plaque. Ann Vasc Surg 2006;20:167-74

51. Rundek T, Arif H, Boden-Albala B, et al. Carotid plaque, a subclinical precursor of vascular events: the Northern Manhattan Study. Neurology 2008;70: 1200-07. Epub 2008 Mar 19

52. Huang D, Swanson E, Lin C, et al. Optical coherence tomography. Science 1991;254:1178-81

53. Yabushita H, Bouma B, Houser S, et al. Characterization of human atherosclerosis by optical coherence tomography. Circulation 2002;106:1640-45

54. Jang I, Bouma B, Kang D, et al. Visualization of coronary atherosclerotic plaques in patients using optical coherence tomography: comparison with intravascular ultrasound. I Am Coll Cardiol 2002;39:604-09

55. Tearney G, Yabushita H, Houser S, et al. Quantification of macrophage content in atherosclerotic plaques by optical coherence tomography. Circulation 2003;107:113-19

56. Kume T, Akasaka T, Kawamoto T, et al. Measurement of the thickness of the fibrous cap by optical coherence tomography. Am Heart J 2006;152:755-55

57. Virmani R, Burke A, Farb A, et al. Pathology of the unstable plaque. Prog Cardiovasc Dis 2002;44:349-56

58. Standish B, Lee K, Jin X, et al. Interstitial Doppler optical coherence tomography as a local tumor necrosis predictor in photodynamic therapy of prostatic carcinoma: an in vivo study. Cancer Research 2008;68:9987-95

59. Fiorella D, Lylyk P, Szikora I, et al. Curative cerebrovascular reconstruction with the Pipeline embolization device: the emergence of definitive endovascular therapy for intracranial aneurysms. J Neurointerv Surg 2009;1:56-65

60. Madjid M, Zarrabi A, Litovsky S, et al. Finding vulnerable atherosclerotic plaques: is it worth the effort? Arterioscler Thromb Vasc Biol 2004;24:1775-82. Epub 2004 Aug 12 